\title{
Design of Home Automation System Using IOT and SMS
}

\author{
Ajagun Abimbola Susan ${ }^{1}$, Awe, Toluwashe Samuel ${ }^{1 *}$ \\ ${ }^{I}$ Department of Electrical and Electronics Engineering, Federal University of Technology Minna, Nigeria.
}

*Corresponding Author: Awe T.S, Department of Electrical/Electronic Engineering, Federal University of Technology, Minna, Niger State, Nigeria.

\begin{abstract}
Humans regularly face security and economic challenges and this is more pronounced in our present society due to increase in crime rate such as housebreaking. Therefore, this project aims at solving this challenge by providing a monitoring and controlling device that alerts its user regardless of its location via Internet and SMS notification for security purposes. This work has five sections: Power Supply Unit, Controller Unit, Connectivity Unit, Relay Unit and Sensing Unit. The Power Supply Unit provides $12 \mathrm{~V}$ and $5 \mathrm{~V}$ to the electromechanical Relay Unit and Controller Unit, the Controller Unit composed of the Arduino Nano controls all functions including relays, sensors and Connectivity Unit; the Connectivity Unit connects the device to the Internet making sure that the Relay Unit is easily controlled via the Internet for turning ON or OFF devices. PIR Sensors and DHT11 are used to monitor motion, average temperature and humidity of the home. The prototype was tested in different locations to ascertain its efficiency and the result obtained showed greater efficiency at locations with highest signal strength.
\end{abstract}

Keywords: Automation system, IOT, SMS, PIR, DHT

\section{INTRODUCTION}

Man's drive for technological advancement has been fueled by his desire to not only access, monitor and control, but to also regulate the amount of energy consumed (to reduce cost and conserve energy) in his immediate environment. This immediate environment is essentially his home, and his needs inadvertently leading to the rise of Home Automation Systems (HAS) for the control of home appliances. Home Automation System (HAS) remains an open and nascent field, though it has been around for a while dating back to the early 1970's but remained an unattained vision due to high costs involved and lack of well-developed technology needed for the realization of the HAS until the late 1970's [1].

Home Automation Systems cannot be constructed without the use of monitoring and control console that also serve as the remote-control access; example of these consoles includes PCs (Personal Computers), mobile phones and other android devices, communication protocols and technologies are also needed such as Bluetooth, ZigBee, Z-Wave, Arduino, Internet and others. Communication techniques are divided into Wire and Wireless connections [2] although wireless technology is more preferred. The advent of the GSM (Global System for Mobile Communication) and subsequently Android Devices has solved the problem of immobility faced due to PCs usage as the monitoring and control console while also providing the services of built-in internet services, Short Message Service (SMS), Wi-Fi (Wireless Fidelity) and Bluetooth wireless technology.

The aim of this design is to develop a HAS to monitor/control the home appliances remotely using various android devices connected to the system and to protect the environment from fire hazards and unwanted intrusion by strangers. Its objectives include:

- To provide enabled users the option to either switch ON or OFF desired appliances through their android devices easily via Web Browser.

- To construct a system that monitors the environment of fire hazards through the use of humidity temperature sensors.

- To construct a device that alerts users of the impending dangers in their environment via SMS.

International Journal of Research Studies in Electrical and Electronics Engineering (IJRSEEE) Page | 10 


\section{REVIEW OF RELATED WORK}

Home Automated System (HAS) using Wi-Fi technology for the monitoring of a variety of sensors and control of appliances was proposed in (1). In this research work, the HAS layout is divided into three sections: the server, hardware interface module and software package. The server uses the Wi-Fi technology for communication between the server and the user and the server and hardware interface module, it is however possible for remote user to connect to the server if the server is connected to the Internet. The hardware interface module is primarily based on Arduino Microcontroller and Wi-Fi Module, software package of this system comprises of the web-based application accessed via a dedicated IP address system on a PC serving as server and the Arduino sketches for control and monitoring of appliances. This model provides a low cost and scalable means of controlling home appliances but depends greatly on the use of repeaters in case of extension over a large coverage area thereby increasing the effective cost.

In 2016, HAS using IOT (2) focuses on the use of renewable energy sources, in this case solar energy, solar energy is used to power the appliances involved. Generally, the system developed can be divided into Hardware unit and Software unit. The Hardware unit consists of the Raspberry Pi, sensors (PIR, Temperature and Float) and actuators, the raspberry pi functions as a PC and is responsible for the interconnectivity of the sensor to the web page my SQL (also serves as GUI), android app was built on a Samsung Mobile is used as remote control to monitor home appliances. The android app, Raspberry's Raspbian OS and My SQL are the software unit of the system. LDRs were also used to track the exact location of the sun in order to generate the highest amount of power possible at any instant. This research strength lies in the use of solar energy for powering but severely limited by the cost of Raspberry Pi and scalability.

The use of Bluetooth as communication protocol of HAS been proposed in (3), this system used the PIC18F2550 due to its serial and USB features. The system was divided into two units Software Design and Main Control Board; software design presented the different approaches of turning ON or OFF any appliance and these include Low Voltage Activating switches (push button), Window GUI and Android GUI. The Main Control Board comprised of the PIC18F2550, relays, USB and Bluetooth Module- Blue Bee necessary for communicating with and control of home appliances. While this system provides redundant systems in the use of USB interface and Low Voltage Activating Switches in the Main Control Board, the main communication technique which is the Bluetooth is limited to 100 meters and therefore this application may not be suitable for large coverage area.

In (4), a HAS that is SMS based through the use of Arduino Uno and GSM Module (SIM 800) was designed. Home appliances are controlled via the use of SMS from a pre-registered user number; the system was divided into two sections, Control Section and Software Section. The Control Section monitors the surrounding for faulty parameters as preset by the user through the use of modules and sensors; the Software Section serves as the remote-control console. This system provides security measures against fire incidents and provides easy access to control home appliances but is severely limited due to overreliance on the signal strength at a particular period of time.

Thus, a Home Automation System using Internet connection for control and SMS for alerting is developed in this research work with a web page serving as Graphic User Interface (GUI) accessed via web browsers (Opera Mini, UC Browser, and Apple's Safari) used as remote control access medium.

\section{Materials And Methods}

HAS is generally divided into five (5) sub- units to increase the simplicity and effectiveness of the design process, the sub-units include: Power Supply Unit, Connectivity/Network Unit, Controller Unit, Sensing and Buzzing Unit, Relay Unit. The Block Diagram of the system as shown in Figure 1 depicts the functional arrangement and interconnectivity of the above stated sub-units. 


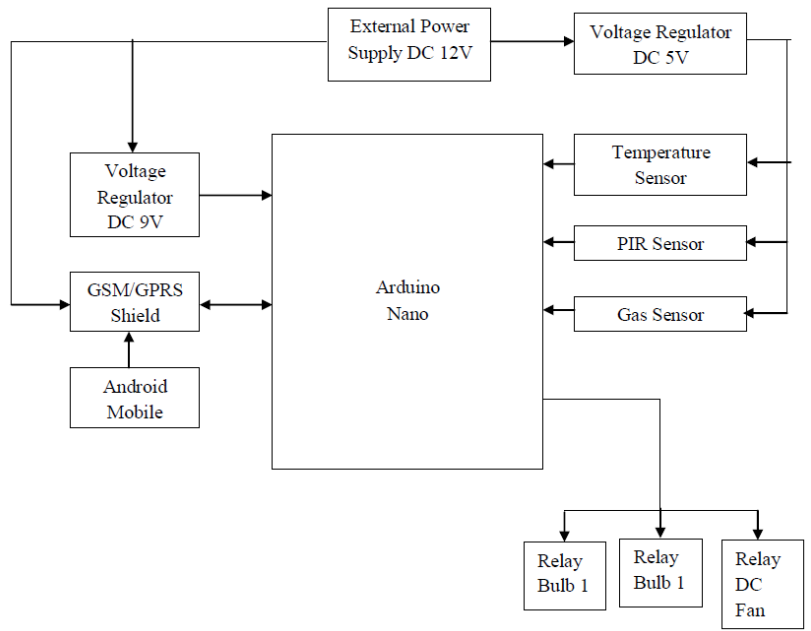

Figure1. Block diagram of the system

\subsection{Power Supply Unit}

The Power Supply Unit consists of a 240/12V step-down transformer, a full bridge rectifier circuit to convert the ac $12 \mathrm{~V}$ to dc $12 \mathrm{~V}$, and the input capacitor with a value of $2200 \mu \mathrm{F}$ is required to suppress any ripple across the voltage regulators, voltage regulators LM 7812 and LM7805 were used to obtain a steady output voltage of $12 \mathrm{~V}$ and $5 \mathrm{~V}$ respectively, output capacitors required to minimize any glitch or spike in output voltage due to transient change in the ac input with a typical value of $10 \mu \mathrm{F}$ were also used. Diodes are used for reverse polarity protection to protect against back EMF. The circuit diagram is depicted in Figure 2 below:

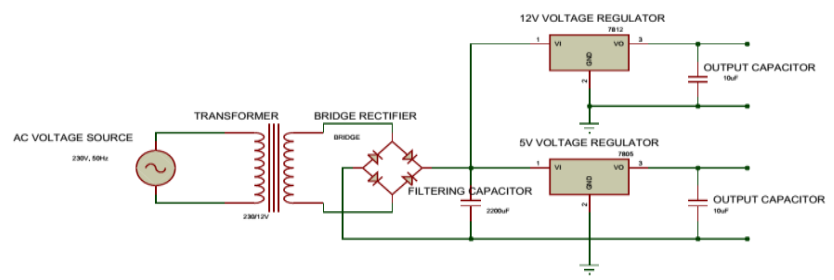

Figure2. Power Supply Unit

\subsection{Connectivity/Networking Unit}

This unit primarily consists of the Arduino Nano, GSM/GPRS Module and the Android Device (which can be a mobile phone or tablet), the GSM/GPRS Module is interfaced to the Arduino Nano by connecting the transmission pin of the Arduino to the reception pin of the GSM/GPRS module and then the reception pin of the Arduino to the transmission pin of the GSM/GPRS module. The GSM/GPRS Module is powered by the external power supply of 5V. The HAS webpage is accessed via the android device and serves as the IOT interface between the internet capability of the GSM/GPRS module and the android device, the webpage depicts vital information such as the humidity, temperature and harmful gas levels and also serve as the remote control via the Internet. The circuit diagram depicting the connectivity unit is shown in Figure 3 below.

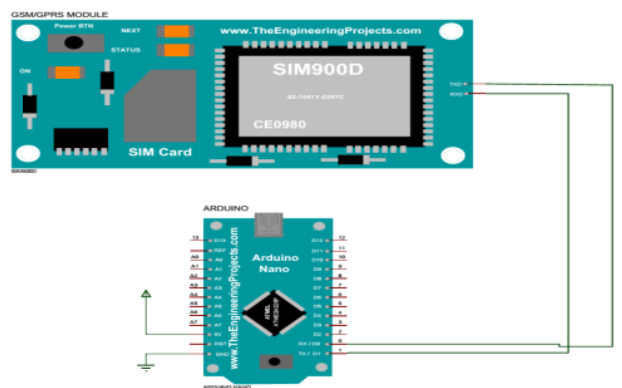

Figure3. Connectivity Unit 


\subsection{Controller Unit}

The controller unit is the Arduino $\mathrm{Na}$ no like the Arduino Uno operates on $5 \mathrm{~V}$ with a voltage range between 6- $20 \mathrm{~V}$ but advised limit of 7-12V. The Arduino Nano is a user-friendly, small and complete board based on microcontroller AT mega 328 and ATmega168, Arduino Nano differs from Arduino Uno in the absence of DC Power Jack with a clock speed of $16 \mathrm{MHz}$ and $16 \mathrm{~KB}$ (for AT mega 168) or $32 \mathrm{~KB}$ (for AT mega 328) flash memory with 14 digital I/O pins and 6 analogue pins; the function of the Arduino Na no is to control the circuit operation by turning ON or OFF the corresponding pins and connecting to the webpage, Arduino Nano is shown in Figure 4 below.

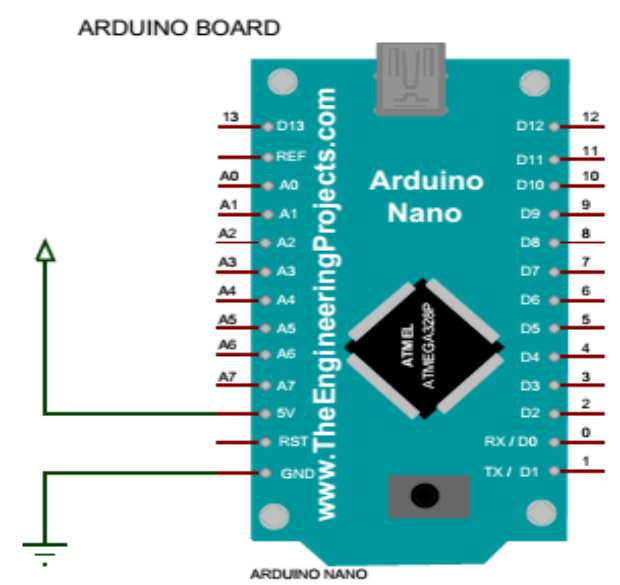

Figure4. Controller Unit- Arduino Nano

\subsection{Sensing Unit}

Sensing units majorly consists of two different sensors which is PIR (Passive Infrared) sensor to detect movement and humidity and temperature sensor to detect high temperatures. The sensing unit serves as the eye and ear of the user even when absent from the home alerting the user via SMS in case of an emergency. Sensing Unit is depicted in Figure 5.

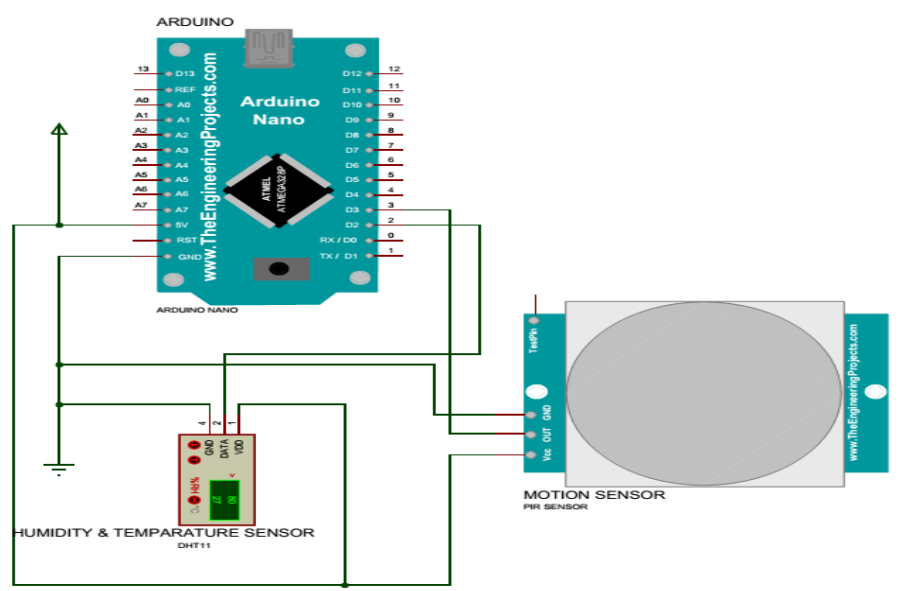

Figure5. Sensing Unit

\subsection{Relay Unit}

Relay unit consists of relays, diodes and transistors; the NPN transistor used is connected through a $1 \mathrm{~K}$ resistor (as the transistor is operated in the saturation region) to the Arduino Nano. Diodes are also used in this configuration to protect the transistors against back EMF, the relays then turn ON or OFF the light bulbs and DC fans. The relays used are of $12 \mathrm{~V}$ ratings with resistance value of $400 \Omega$, transistor used is 2N2222 which is of Low Power, Medium Current and Medium Voltage ratings and is commonly used for switching action. The relaying arrangement is show in Figure 6. 


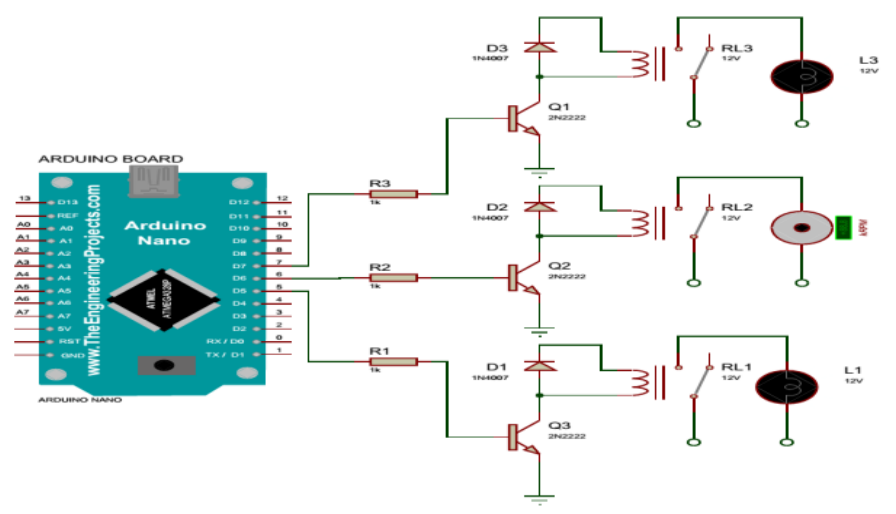

Figure6. Relay Unit

\section{TEST, RESULT AND DISCUSSION}

\subsection{Test and Result}

The design was implemented, tested unit by unit and on the whole system. The results of each test are outlined in this section. The discussion of result is also presented.

\subsubsection{Power Supply Unit}

The input voltage from the ac mains supply was measured to ascertain that the value is neither too high nor too low. The input wires of the step-down transformer were connected to the mains supply and the output ac voltage was observed and duly noted. The Multimeter knob was carefully switched for appropriate DC value measurement, polarity was ascertained and the voltages across bridge rectifier and voltage regulators were measured and noted in Table 1.

Table1. Voltage Results obtained from Power Supply Unit Test

\begin{tabular}{|c|c|c|c|}
\hline Component & Input (V) & Output Obtained(V) & Expected Value (V) \\
\hline Transformer & 230 & 12.69 & 12.00 \\
\hline Bridge Rectifier & 12.69 & 16.65 & 15.57 \\
\hline LM7805 & 16.65 & 12.22 & 12.00 \\
\hline LM7812 & 16.65 & 5.00 & 5.00 \\
\hline
\end{tabular}

\subsubsection{Connectivity/Networking Unit}

This unit is primarily saddled with the responsibility of connecting the HAS to the Web page for control of the home appliances. All interconnections were checked carefully to make sure the connections were right and the Arduino and SIM 800L data sheets were used to confirm the ratings. The voltage input to the GSM/GPRS module from the external power source was measured as shown in Table 2. Connectivity to the Webpage was tried ten times and the ratio of successful connections to total trial times was calculated.

Table2. Voltage and Current results measured for GPRS/GSM Shield

\begin{tabular}{|c|c|c|c|c|}
\hline Component & $\begin{array}{c}\text { Expected Current } \\
\text { Value (A) }\end{array}$ & $\begin{array}{c}\text { Expected Voltage } \\
\text { Value (V) }\end{array}$ & $\begin{array}{c}\text { Voltage } \\
\text { Obtained (V) }\end{array}$ & $\begin{array}{c}\text { Current } \\
\text { Obtained (A) }\end{array}$ \\
\hline GSM/GPRS & 2.00 & $3.5-4.5$ & 3.7 & 2.30 \\
\hline
\end{tabular}

Reliability of Connectivity Unit, $\boldsymbol{R}_{\boldsymbol{C U}}=\frac{\text { Number of successful connections }}{\text { Total number of trials }}$

Number of successful connection $=8$

Total number of trials $=10$

$\boldsymbol{R}_{C U}=\frac{8}{10}=0.8$ or $80 \%$

Figure 7 shows the result obtained when connectivity is successful, the web page depicted below pops up and provides the user with virtual switches that can then be used to turn ON/OFF home appliances as required, this is shown in Figure 8. 


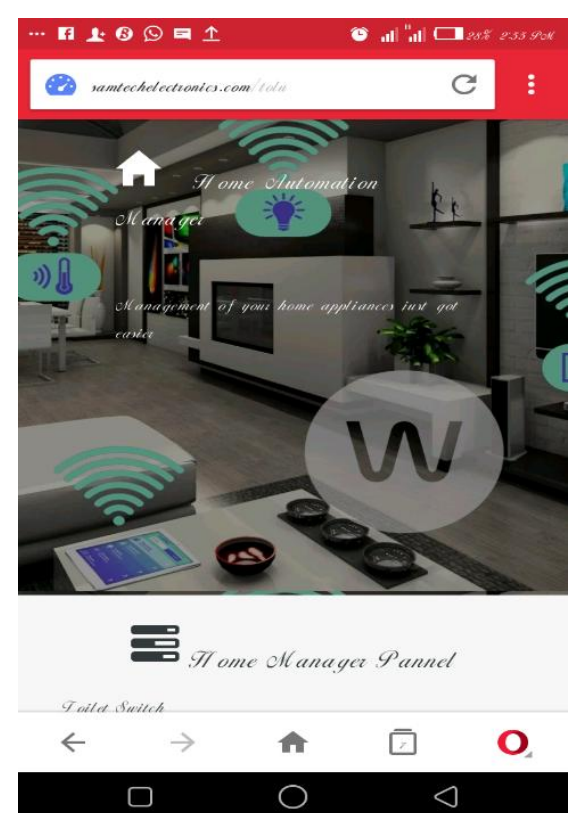

Figure7. Home Page of Home Automation System

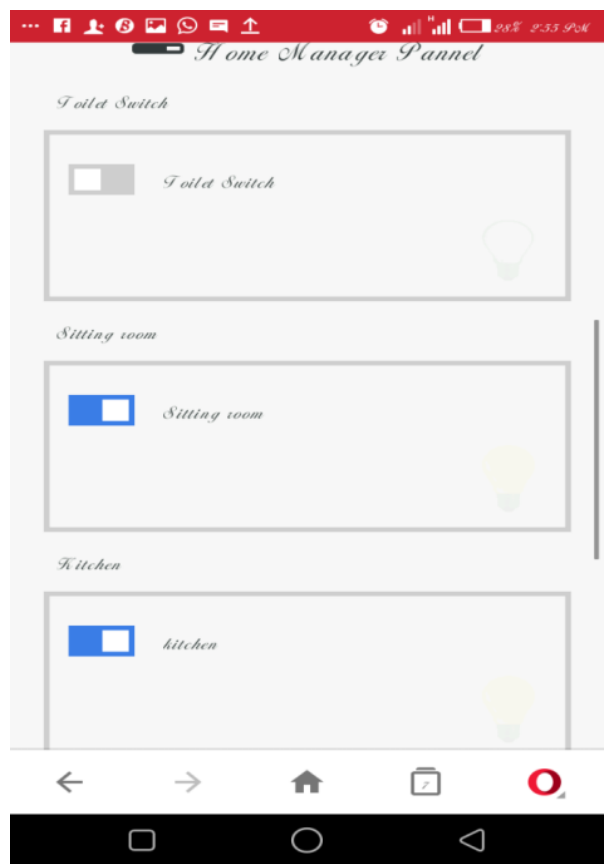

Figure8.Virtual Switches to control home appliances webpage

\subsubsection{Sensing Unit}

This unit is comprised of PIR Motion sensor and DHT11 Sensor and is required for the monitoring of the HAS. The PIR Motion Sensor and DHT11 sensor data sheet was used to confirm the required ratings needed and then the circuit connections were confirmed.Using the Multi-meter the output voltages and input voltages of the PIR Motion and DHT11 Sensor. The Sensitivity of the PIR Motion and DHT11 sensor was tested by altering the distance and temperature respectively and the delay time is measured for both sensors via timer and duly noted in Table 3 and Table 4.

Table3. PIR Motion Sensor Result Data

\begin{tabular}{|l|c|c|c|c|}
\hline Data & Input Voltage (V) & Output Voltage (V) & $\begin{array}{c}\text { Maximum } \\
\text { Sensitivity (m) }\end{array}$ & Delay (s) \\
\hline $\begin{array}{l}\text { Manufacturer's } \\
\text { Data }\end{array}$ & $3-6$ & 5 or 0 & 9 & $4-30$ \\
\hline $\begin{array}{l}\text { Measured } \\
\text { Quantities }\end{array}$ & 5 & 4.89 or 0 & 7 & $5-32$ \\
\hline
\end{tabular}


Table4. DHT Sensor Result Data

\begin{tabular}{|c|c|c|c|}
\hline Data & Input Voltage (V) & Output Quantities & Delay (s) \\
\hline Manufacturer's Data & $3-5.5$ & $20-90 \%$ RH, 0- 50 $\mathrm{C}$ & $6-30$ \\
\hline Measured Quantities & 5 & $20-90 \%$ RH, 0- 50 $\mathrm{C}$ & $7-32$ \\
\hline
\end{tabular}

- The Reliability of the PIR Motion and DHT sensors were calculated by taking the ratio of successful trials to total number of trials.

Reliability of Sensor Unit, $\boldsymbol{R}_{\boldsymbol{S U}}=\frac{\text { Number of successful sensing }}{\text { Total number of trials }}$

Number of successful sensing $=4$

Total number of trials $=5$

$\boldsymbol{R}_{\text {SU }}=\frac{4}{5}=0.8$ or $80 \%$

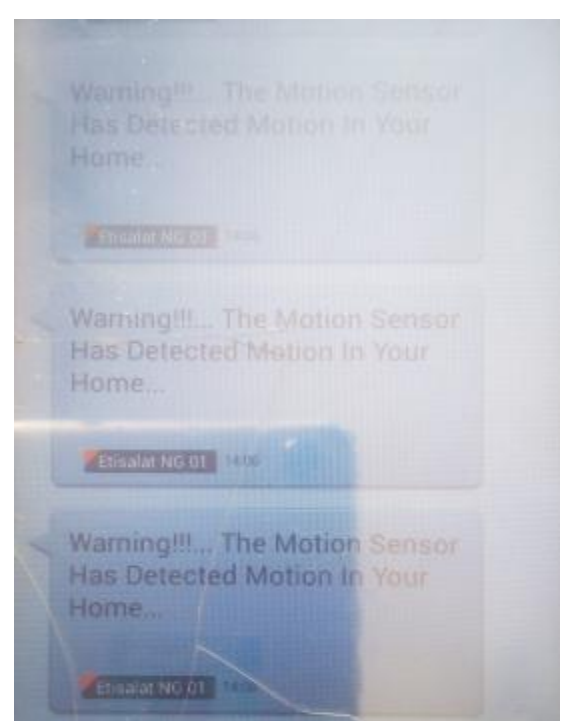

Figure9. Result of SMS from PIR sensor

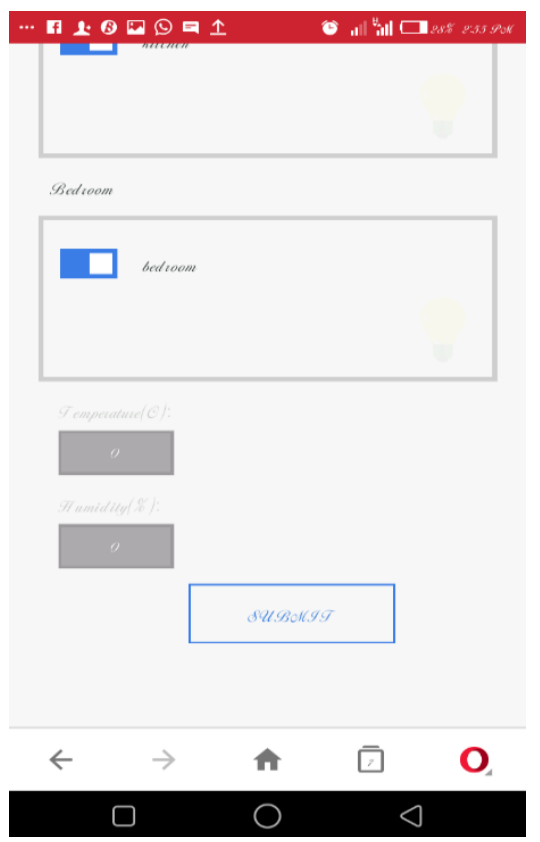

Figure10.Result from DHT Sensor when device is in OFF mode

\subsubsection{Relay Unit}

This sub-section involves test performed on the relay units which comprises of diodes, transistors and the relay itself. The voltages and currents across the diodes were measured, the transistors input 
voltages and currents were measured and the input voltage and output voltage to the relay is measured. This is shown in Table 5 and 6.

Table5. Relay Unit Voltage Test Result

\begin{tabular}{|c|c|c|c|c|}
\hline Component & $\begin{array}{c}\text { Expected Input } \\
\text { Voltage (V) }\end{array}$ & $\begin{array}{c}\text { Measured Input } \\
\text { Voltage (V) }\end{array}$ & $\begin{array}{c}\text { Expected Output } \\
\text { Voltage (V) }\end{array}$ & $\begin{array}{c}\text { Measured Output } \\
\text { Voltage (V) }\end{array}$ \\
\hline Transistor & 12 & 13 & 23 & 27 \\
\hline Diode & 4 & 7 & 3 & 5 \\
\hline Relay & 8 & 9 & 12 & 8 \\
\hline
\end{tabular}

Table6. Relay Unit Current Test Result

\begin{tabular}{|c|c|c|c|c|}
\hline Component & $\begin{array}{c}\text { Expected Input } \\
\text { Current (A) }\end{array}$ & $\begin{array}{c}\text { Measured Input } \\
\text { Current (A) }\end{array}$ & $\begin{array}{c}\text { Expected Output } \\
\text { Current (A) }\end{array}$ & $\begin{array}{c}\text { Measured Output } \\
\text { Current (A) }\end{array}$ \\
\hline Transistor & 34 & 23 & 45 & 18 \\
\hline Diode & 56 & 45 & 44 & 65 \\
\hline Relay & 12 & 45 & 44 & 33 \\
\hline
\end{tabular}

- The Delay time of the relay is then tested by applying voltage across the relay; the time was measured and noted.

- The reliability of the Relay unit was calculated by taking the ratio of successful trials to total number of trials.

Reliability of Relay Unit, $\boldsymbol{R}_{\boldsymbol{R} \boldsymbol{U}}=\frac{\text { Number of successful actuations }}{\text { Total number of trials }}$

Number of successful actuations $=9$

Total number of trials $=10$

$\boldsymbol{R}_{\boldsymbol{R} U}=\frac{9}{10}=0.9$ Or $90 \%$

\subsubsection{HAS}

The HAS itself as a unit is tested by checking the functionality of the overall parts, to confirm that the achieved purposes of the HAS had being met.

- After connecting to the webpage, the home appliances were energized and de-energized and the time delay between energizing/de-energizing on webpage and the actual actuating was measured and recorded for each home appliance in Table 7.

- The PIR Motion and DHT11 sensors were energized and the time delay before SMS is received for each mobile device was measured, noted and recorded in Table 7.

- To calculate the system's reliability, the Sensing Unit reliability and the Relay unit reliability was utilized through equation 4.4, Where R denotes reliability.

$\boldsymbol{R}_{\boldsymbol{H A S}}=1-\left(1-\boldsymbol{R}_{\boldsymbol{R U}}\right)\left(1-\boldsymbol{R}_{S U}\right)\left(1-\boldsymbol{R}_{\boldsymbol{C U}}\right)$

$\boldsymbol{R}_{\text {HAS }}=1-(1-0.8)(1-0.8)(1-0.9)$

$\boldsymbol{R}_{\text {HAS }}=0.996$ or $99.6 \%$

Table7. HAS Delay Time Test Result

\begin{tabular}{|c|c|}
\hline Component & Delay Time (s) \\
\hline Lamp 1 & 12 \\
\hline Lamp 2 & 13 \\
\hline DC Fan & 12 \\
\hline PIR Motion Sensor & 14 \\
\hline DHT11 Sensor & 8 \\
\hline
\end{tabular}

4.1.5. Operational Flow Chart

The flow chart of the HAS in Figure 7 is a pictorial algorithm depicting the flow/ logical operation of the system. The system starts operating by the initialization of the HAS while the user's android 
mobile assumed to be in OFF state initially is turned ON, HAS instantly measures humidity and temperature parameters of the surrounding and updates the webpage if service is available. The webpage is continually updated until the user energizes or de-energizes one or more relays via the webpage which is instantly used to turn ON/OFF the respective device actuated, while doing this the PIR Sensor and DHT11 continues to monitor

the surrounding, in case the value measured at a certain instant, $\mathrm{T}$, is greater than maximum temperature, $\mathrm{T}_{\mathrm{m}}$ and/or movement is detected, the HAS instantly sends a message to the User's designated android phone number.

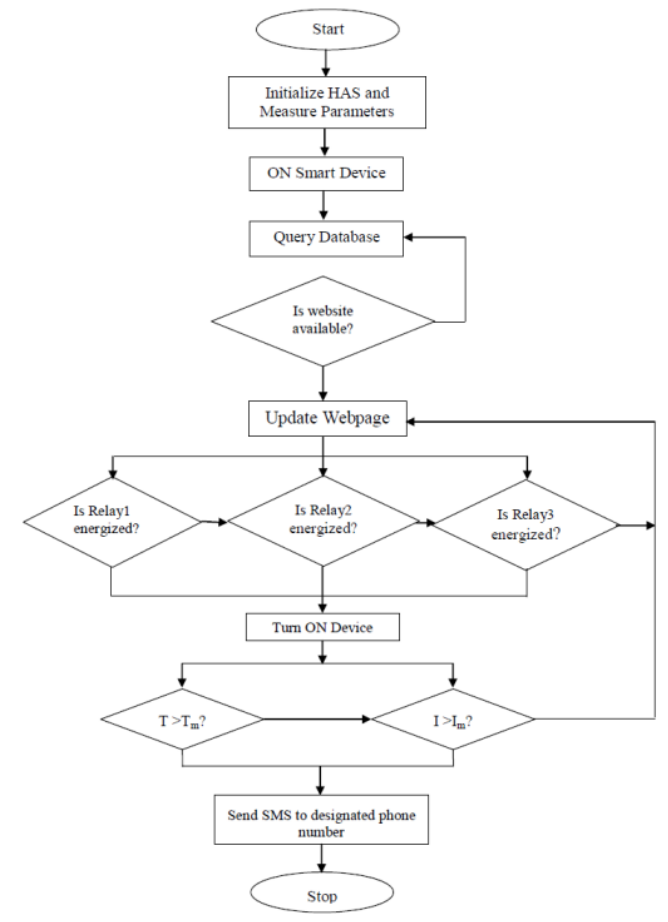

Figure11. Flow Chart depicting the working operation of the Home Automation System (HAS)

\subsection{Discussion of Result}

The Result obtained as shown in Figure 7 and Figure 8 showed that the Home Automation System is capable not only of controlling home appliances via the Internet with the website www.samtechelectronics.com/tolu serving as the GUI but also capable of monitoring the environment as shown in Figure 9, Figure 10 also proves that the HAS is capable of alerting the user via SMS. The performance of each and every unit in this project was analyzed and obtained as $0.8,0.8$ and 0.9 for controller unit, sensing unit and relay unit respectively leading to a total reliability of 0.996 for the system as whole. Therefore, the aim and objectives of this project were achieved that is to design and construct a device capable of monitoring/controlling home appliances using IoT and SMS.

\section{CONCLUSION AND FUTURE WORK}

\subsection{Conclusion}

This work "Android Home Automation System using IOT" involves the control of Home Appliances via the Internet with webpage as GUI, PIR Motion Sensor and DHT Sensor. The Android HAS using IOT eases control of home appliances, helps in energy conservation and aid in protection of lives and properties of the aged, sick and in fact all and sundry. It also serves as Security and Fire Hazard aversion devices to protect the aged and sick via SMS.

\subsection{Future Work}

The home automation though still rapidly growing has lots of closed doors to explore depending on the taste of the end-user and the engineer/technician involved. During the course of this project the use of the IOT capability embedded in all mobile devices as GSM/GPRS connectivity protocols to connect the user to the Internet, web pages was developed via the use of php web programming language and connectivity to the HAS was accomplished through the use of SIM800. Actuation home 
appliances was simply achieved through the use of electromechanical relays in the HAS via the webpage. For future work, the following is recommended;

- Electromechanical relays should be replaced with solid state relays, this is due to the fact that while electromechanical relays are also effective, it consumes more energy than solid state relays.

- The number of PIR Motion sensor used should be increased in areas that are vulnerable to theft and housebreaking, furthermore, DHT sensors should also be increased in areas that are liable to fire hazards.

- In case measured temperature is above a certain limit, it should be able to send messages to a designated phone number.

- Better secured web pages should be used in future applications in order to enhance security of the home from outside invasion.

- The GSM/GPRS shield if available should be $3 \mathrm{G}$ or $4 \mathrm{G}$ in order to increase communication quality between the user and the home.

\section{REFERENCES}

[1] A. Elshafee and K. A. Hamed, "Design and Implementation of a Wi-Fi Based Home Automation System," World Academy of Science, Engineering and Technology, vol. 6, November 2012.

[2] D.D. Ghorpade and A.M. Patki, "A Review on IOT Based Smart Home Automation Using Renewable Energy Sources", International Journal of Science and Research (IJSR), vol. 5, no. 5, May 2016

[3] R. A. Ramlee, M. H. Leong, R. S. Singh, M. M. Ismail, M. A. Othman, H. A. Sulaiman, M. H. Misran and M. A. Meor Said, "Bluetooth Remote Home Automation System Using Android Application," The International Journal of Engineering and Science, vol. 2, no. 1, pp. 149-153, 2013.

[4] A. Patil, P.Potnis and K.Katkar, "SMS Based Home Automation System Using Android ATMEG328 with GSM", International Journal of Engineering Trends and Technology (IJETT), vol. 47, no. 7, pp. 369-374, May 2017.

[5] L. Haddon, "Home Automation: Research Issues," in 2nd EMTEL Workshop: The European Telecom User, Amsterdam, Netherlands, 1995.

Citation: Ajagun Abimbola Susan \& Awe, Toluwashe Samuel "Design of Home Automation System Using IOT and SMS", International Journal of Research Studies in Electrical and Electronics Engineering, 4(2), pp 10-19. DOI: http://dx.doi. org/10.20431/2454-9436.0402002

Copyright: () 2018 Authors. This is an open-access article distributed under the terms of the Creative Commons Attribution License, which permits unrestricted use, distribution, and reproduction in any medium, provided the original author and source are credited. 